\title{
Evaluation of Memory Complaints in People Living with HIV in Iran
}

\author{
Malihe Sheibani ${ }^{1,{ }^{*}}$, Nurallah Mohammadi ${ }^{1}$, Hassan Joulaei $(\mathbb{D})^{2}$ and Zohre Foroozanfar (id ${ }^{2}$ \\ ${ }^{1}$ Department of Clinical Psychology, Shiraz University, Shiraz, IR Iran \\ ${ }^{2}$ Shiraz HIV/AIDS Research Center, Institute of Health, Shiraz University of Medical Sciences, Shiraz, IR Iran \\ "Corresponding author: Department of Clinical Psychology, Shiraz University, Shiraz, IR Iran. Email: msh.psych65@yahoo.com
}

Received 2021 October 09; Revised 2021 November 13; Accepted 2021 November 14.

\begin{abstract}
Background: Several studies have reported that many people living with HIV (PLHIV) complain of memory impairments. Objectives: This study aimed to investigate these complaints both subjectively and objectively.

Patients and Methods: For this purpose, 50 PLHIV and 50 of their companions as the control group were selected by the convenience sampling method in Shiraz, Iran, and completed Ray's auditory-verbal learning test and the Patient's Assessment of Own Functioning Inventory (PAOFI). Data were analyzed using a $t$-test in SPSS.

Results: Our findings showed a significant difference between PLHIV and the control group in terms of subjective memory complaints and objective memory functions.

Conclusions: The overall result of the study showed that PLHIV reported more subjective memory complaints and poorer objective memory functions than the control group, regardless of the stage of the disease.
\end{abstract}

Keywords: Subjective Memory Complaints, Objective Memory Function, HIV, Iran

\section{Background}

One of the most important diseases that has created many challenges for patients and health care systems both physically and psychologically is HIV infection. Unfortunately, the incidence of HIV infection is increasing in Iran. About 4,100 new infections occur each year, and 2,500 people die from the disease (1). According to the latest data until the end of 2019, the total number of registered HIV infected people was 41,494 , of whom $82 \%$ were men and $18 \%$ were women. The main reasons for HIV incidence are injection with shared needles followed by sexual intercourse. However, in recent years, sexual intercourse has accounted for a higher percentage of HIV-positive cases (2).

People living with HIV (PLHIV) face many limitations in the social and cultural environment. These people have experiences that are often stressful (3). A wide range of disease processes may affect the brain of an HIV-infected person. Although a key feature of HIV infection is the gradual decline in the body's ability to show the appropriate immune response, neuropsychological phenomena are also evident. More than 50\% of people with this infection have neuropsychiatric manifestation, which is the first sign of the disease in $10 \%$ of HIV-positive cases (4). Another sign of the disease is cognitive impairment. PLHIV shows several cognitive impairments, from mild motor-cognitive impairment to HIV dementia (5). Some HIV patients report low performance in executive function, information processing speed, working memory, verbal learning, delayed recall, recognition, attention, and psychomotor speed despite receiving highly active antiretroviral therapy (6-9).

Some studies on memory functions $(10,11)$ have shown that in HIV negative and positive people, the function of memory is different. They examined memory function both subjectively and objectively. Subjective memory complaints are self-reported complaints that may reflect objective cognitive impairment. The prevalence of these complaints is high in old age (more than 50\%) and includes distraction, forgetfulness, and loss of consciousness (12). Factors such as poor daily functions, personality traits, nervous system problems, or mood disorders such as anxiety, depression, and even insomnia may be the cause of these complaints $(10,12)$. These complaints may be reported in both men and women of any age and increase during the illness (12). The report of subjective memory complaints may predict cognitive impairment in later stages of the disease. Thus, these complaints are considered essential for examining the trend of cognitive changes in PLHIV $(11,13)$. These complaints are significant in the early stages of the disease because it is challenging to diagnose neuropsychological damage in these stages $(14,15)$.

Unfortunately, these issues are often ignored in the 
care of PLHIV. Neurological and psychiatric disorders may impair the ability to understand and follow appropriate treatment programs for these patients and directly lead to low adherence to antiretroviral therapies (16).

\section{Objectives}

The majority of studies on neuropsychological disorders associated with HIV have been conducted in developed countries. Thus, this study examined subjective memory complaints and objective memory functions in PLHIV and the control group.

\section{Patients and Methods}

\subsection{Research Plan and Sampling}

Our study was based on a comparative causal design between PLHIV and healthy people. The independent variable was the type of group, and the dependent variables were subjective memory complaints and objective memory functions. Thus, based on previous studies, 50 patients and 50 of their companions above 18 were selected using convenience sampling from the total PLHIV admitted to the Shiraz Voluntary Counselling and Testing Center (VCT). This center is a referral center for all HIV-positive patients living in Fars Province, Iran. In this center, 3714 HIV positive cases have been registered from 2003, of whom 2386 cases are still alive. However, only 1383 (58\%) of the cases have active files and receive ongoing care. Criteria for exclusion from the sample group were as follows: (1) having an infection in the central nervous system such as MS; (2) head injuries, which lead to anesthesia for more than 1 hour; (3) epilepsy or psychiatric disorders including delirium and 4having no history of substance abuse.

\subsection{Data Collection}

The interview was conducted in a private room in the VCT by a clinical psychologist. After the subjects were provided with necessary explanations about the importance of the research, they were assured that their information would remain confidential. Also, after obtaining consent from the subjects and giving them some money as reward, the measures were administered: (1) Ray's auditoryverbal learning test (Ray, 1964; Sprin \& Strauss, 1991); and (2) the Patient's Assessment of Own Functioning Inventory (PAOFI) (Chelune, Heaton, \& Lehman 1986). For subjects who did not have enough education, the questions were read, and their responses were recorded. How the research measures were implemented is mentioned in the next section.

\subsection{Measures}

\subsubsection{Ray's Auditory-Verbal Learning Test}

The test, which is used to assess the objective function of memory, consists of a list of 15 irrelevant words (list A). The words are repeated five times for the subject, and they are asked to recall it. The subject is then presented with another list of 15 unrelated words (list B) used list A. Next, the subject is asked to recall as many words as they can from the initial list. After a 30-minute delay, the subject is asked to memorize the words from the initial list (list A) and then recognize as many words as they can from another list, including words from the initial list. All the steps take between 10 and 15 minutes. The test's retest validity has been reported to be 0.55 on average for one year (17). In Iran, the test's convergent validity was obtained at the rate of $(\mathrm{r}=0.346)(\mathrm{P}<0.001)(18)$. Various lists of this test have been compiled, and a potential problem with the test is that no specific instructions have been published on interpretation and application. Some authors recommend 20 minutes and others 30 minutes between the first and second tests. In this study, Lezak's instructions were more reliable as it has been frequently used in most studies. Also, the distance between the two tests was considered $20 \mathrm{~min}$ utes in this study. The delayed recall was used for objective memory functions.

\subsubsection{The Patient's Assessment of Own Functioning Inventory (PAOFI)}

It is a self-reported questionnaire based on which respondents rate how they experience specific problems in various areas of cognitive function, including memory, language and communication, sensory perception and motor skills, and higher-level cognitive functions. The questionnaire has 33 questions in four subscales, including memory (10 questions), language and communication (nine questions), sensory perception and motor skills (five questions), and higher-level cognitive and intelligence functions (nine questions). Participants are asked to rate their memory problems on a 6-point Likert scale $(0=$ almost never; 1 = very infrequently; 2 = once in a while; $3=$ fairly often; 4 = very often; 5 = almost always). Subjects' scores in each of the subscales are added together and divided by the number of questions in the same subscale. In fact, for each subscale, an average score is obtained. In the study of Chan et al. (2007), PAOFI's internal consistency ( $\alpha$ $=0.87$ ) was proven (19). We measured PAOFI's validity and reliability in our study. The internal consistency coefficient of the questionnaire was desirable (Cronbach's alpha was 0.93 for the overall questionnaire and 0.86 for the memory subscale). Indicators of confirmatory factor analysis (20) showed relatively good fitness $(\mathrm{GFI}=0.66, \mathrm{NFI}=0.6, \mathrm{RMSEA}$ $=0.09$ ). 


\subsection{Ethical Approval}

This article is derived from a dissertation approved by the Shiraz University in collaboration with the Shiraz AIDS Research Center. The study was approved by the Ethical Committee of the Shiraz University of Medical Sciences with the code number IR.SUMS.REC.1391.2605.

\section{Results}

In PLHIV, 10\% were male, and 90\% were female. The age range of the patients was between 24 and 54 years, with an average of $38.34 \pm 8.41$. The average years of education in the patients were $8.66 \pm 4.08$, about $56 \%$ of them were married, and $84 \%$ of them were unemployed. In the control group, $48 \%$ of the participants were male, and $52 \%$ of them were female. The age range of the participants was between 20 and 50 years, with an average of $33.42 \pm 9.36$. The average years of education in the participants were $8.28 \pm 3.91$. Also, $76 \%$ of the participants were married, and many of the participants were unemployed (70\%) (Table 1). The frequency of subjective memory complaints on the PAOFI-memory is shown in Table 2. Losing things or having trouble remembering where they are and forgetting something told within the last day or two are the most complaints in PLHIV and the control group.

\begin{tabular}{|c|c|c|}
\hline Variables & Control Group $(n=50)$ & $\operatorname{PLHIV}(n=50)$ \\
\hline Age $(y)$ & $33.42 \pm 9.36$ & $38.34 \pm 8.41$ \\
\hline Education $(y)$ & $8.28 \pm 3.91$ & $8.66 \pm 4.08$ \\
\hline \multicolumn{3}{|l|}{ Gender } \\
\hline Male & $24(48)$ & $5(10)$ \\
\hline Female & $26(52)$ & $45(90)$ \\
\hline \multicolumn{3}{|l|}{ Marital status } \\
\hline Single & $8(16)$ & $1(2)$ \\
\hline Married & $38(76)$ & $28(56)$ \\
\hline Divorced & $4(8)$ & $21(42)$ \\
\hline \multicolumn{3}{|l|}{ Occupational status } \\
\hline Employed & $15(30)$ & $8(16)$ \\
\hline Unemployed & $35(70)$ & $42(84)$ \\
\hline
\end{tabular}

The mean score of memory complaints was $10.00 \pm$ 4.70 in the control group and $13.30 \pm 9.28$ in the experimental group. Also, the mean score of delayed recall was $11.52 \pm 2.04$ and $9.98 \pm 2.28$ in the control and experimental groups, respectively (Table 3 ).

We hypothesized that people living with HIV and the control group differed in subjective memory complaints and objective memory functions. In order to test this hypothesis, a $t$-test was used, and the results showed that there was a significant difference between PLHIV and the control group regarding subjective memory complaints ( $P$ $<0.000)$. Also, there was a significant difference between PLHIV and the control group regarding objective memory functions $(\mathrm{P}<0.02)$. People living with HIV reported more subjective memory complaints and less objective memory functions than the control group (Table 3).

\section{Discussion}

This study was implemented on HIV-positive patients for the first time in Iran. HIV-associated cognitive impairments lead to the destruction of cognitive function and reduced mental activity, preventing one from working, housekeeping, or social functioning. They may usually progress to HIV dementia, but timely treatment may inhibit HIV dementia. The current study checked out an essential element of cognitive functions, namely memory. The study examined memory complaints in PLHIV using a preliminary validation of the memory subscale of PAOFI. It has ten questions that evaluate patients' complaints about daily activities. The validity and reliability of PAOFI were desirable. Reviewing the items showed that losing things or having trouble remembering where they are was reported as the most common memory complaint in PLHIV. However, in the control group, forgetting something told within the last day or two had the highest frequency.

There was a significant difference between PLHIV and the control group in terms of reporting memory complaints and memory functions. PLHIV reported more problems in assessing their memory than the control group. This finding is consistent with the findings of Hinckin et al. (1996) showing that HIV positive individuals had more cognitive complaints than HIV negative individuals, regardless of their stage of infection (21). Papirovits et al., 2021, considered subjective memory complaints a key component in mild cognitive impairment (MCI) diagnosis. They showed no significant difference in working memory assessment between the healthy group and those with MCI. MCI participants estimated their semantic memory less than the healthy group and had more episodic memory capabilities. Thus, they suggested that when using selfreport criteria to diagnose MCI, physicians should pay attention to the patient's semantic memory complaints (22). In our study, two factors can be considered to explain findings. The first is that these complaints result from patients' mood swings after being diagnosed with HIV, and the second is that these patients have real memory problems. Regardless of the stage of the disease, the diagnosis of HIV and its associated psychological consequences 


\begin{tabular}{|c|c|c|}
\hline Complaint & Control Group $(\mathbf{n}=\mathbf{5 0})$ & $\operatorname{PLHIV}(\mathbf{n}=50)$ \\
\hline 1. Forget something told within the last day or two & $9(18)$ & $10(20)$ \\
\hline 2. Forget events occurring in the last day or two & $1(2)$ & $4(8)$ \\
\hline 3. Forget people met in the last day or two & $0(0)$ & $5(10)$ \\
\hline 4. Forget things knew a year or more ago & $2(4)$ & $6(12)$ \\
\hline 5. Forget people met a year or more ago & $0(0)$ & $7(14)$ \\
\hline 6. Lose track of time & $6(12)$ & $14(28)$ \\
\hline 7. Fail to finish something started because forgotten it was being done & $0(0)$ & $11(22)$ \\
\hline 8. Fail to complete a task started because forgotten how to do one or more aspects of it & $0(0)$ & $9(18)$ \\
\hline 9. Lose things or have trouble remembering where they are & $5(10)$ & $17(34)$ \\
\hline 10. Forget things supposed or agreed to be done & $1(2)$ & $12(24)$ \\
\hline
\end{tabular}

Abbreviation: PAOF, Patient's Assessment of Own Functioning Inventory.

a Presence of complaints as defined by the endorsement of responses including "Fairly often," "Very often," or "Almost always".

${ }^{\mathrm{b}}$ Values are expressed as No. (\%).

\begin{tabular}{lcccccc}
\hline Table 3. The $t$-test Results in PLHIV and the Control Group $^{\text {a }}$ & \multicolumn{1}{c}{} & & & & \\
\hline Dependent Variable & Control Group & PLHIV & $\boldsymbol{t}$ & df & Mean Difference & \multicolumn{1}{c}{ P } \\
\hline Subjective memory complaints & $10.00 \pm 4.70$ & $13.30 \pm 9.28$ & 2.28 & 98 & 3.3 & 0.02 \\
Objective memory functions & $11.52 \pm 2.04$ & $9.98 \pm 2.28$ & -4.15 & 98 & -1.54 & 0.001 \\
\hline
\end{tabular}

${ }^{\text {a }}$ Values are expressed as mean \pm SD.

such as anxiety, depression, hopelessness, and many other consequences have a significant impact on patients' assessment of their daily functioning in life. The patient group may overestimate their memory problems due to the stress caused by the diagnosis of HIV, especially in our religious society. Akbari et al., 2020, stated that stigma in PLHIV seriously affected their quality of life (23), this is an essential factor that must be considered in assessing patients' daily functioning in future studies.

Another finding, in line with previous studies (7, 11), was a significant difference between the mentioned groups in terms of objective memory functions. Kamkwalala et al., 2016, reported that HIV-negative adults had a higher delayed recall score than HIV positives (11). Maki et al., 2014, showed a lower score on verbal memory in HIV women (7). It is necessary to point out the effect of HIV on patients' cognitive functions to explain this finding. As in the history of HIV, the occurrence of neurological disorders caused by this virus has been proven, and in some cases, these disorders even lead to dementia caused by AIDS. Au and his colleagues (2008) concluded that, in terms of objective performance on the HKLLT memory test, mild memory impairment was seen between $13.2 \%$ and $28.9 \%$ of participants. The delayed recall was mostly damaged (9). Dawes et al., 2008, stated that a weakened immune system and the progression of the disease was often associ- ated with neurological damage, but the damage pattern was variable (24). Therefore, it seems that the effect of HIV on patients' memory is definite, and it is necessary to determine the effect of this factor and other factors affecting the memory of these patients. According to the study results, it is appropriate to consider subjective memory complaints in developing treatment plans for PLHIV and determine whether these complaints are related to HIV infection. One of the significant challenges in developing effective treatment is to differentiate the effects of the disease from the effects of common comorbidities, including emotional disorders such as depression and anxiety.

\subsection{Limitations}

Among the executive limitations of the study were the time-consuming nature of the study and the two-stage nature of Ray's test; in case of the latter, the patients sometimes did not refer for the second stage, and the researcher had to remove them from the sample. Another limitation was that we conducted this study on patients who were not addicted to drugs or alcohol, while an impressive number of these patients are addicted to drugs and have been infected by co-injection. In future studies, this variable needs to be considered. On the other hand, since we gave each participant some money as reward, financial constraints 
prevented us from increasing the sample size. Future studies should examine a larger sample to have more generalizability.

\subsection{Conclusion}

Overall, people with HIV reported more subjective memory complaints than the control group, while the control group showed better objective memory functions. Some strategies should be used to prevent the occurrence of these injuries in HIV-positive patients. The possibility of comparing the variables in addicted and non-addicted HIV-infected people is one of the most critical and fundamental suggestions of this study. Also, other cognitive complaints raised in PAOFI should be evaluated, and the accuracy of these complaints should be assessed using a wide range of neuropsychological tools. Finally, other variables, such as length of time since HIV infection, type of drug used in addicted patients, and anxiety, can also be examined in future research.

\section{Acknowledgments}

This article is part of a dissertation approved by the Shiraz University and was financially supported by the Shiraz University of Medical Sciences (SUMS) in Iran. The authors would appreciate all HIV-positive patients for their participation and also managers of the VCT center of Shiraz for their coordination.

\section{Footnotes}

Authors' Contribution: Malihe Sheibani, planning research, collecting data, and doing statistical analysis; Nurallah Mohammadi, supervising and doing statistical analysis; Hassan Joulaei, co-supervising, reviewing, and editing the manuscript; Zohre Foroozanfar, reviewing and doing statistical analysis.

Conflict of Interests: There is no conflict of interest.

Ethical Approval: This article is derived from a dissertation approved by the Shiraz University in collaboration with the Shiraz AIDS Research Center. The study was approved by the Ethical Committee of the Shiraz University of Medical Sciences with the code number IR.SUMS.REC.1391.2605.

Funding/Support: The Research Deputy for the Shiraz University of Medical Sciences

Informed Consent: The interview was conducted in a private room in the VCT by a clinical psychologist. After necessary explanations were provided to the subjects about the importance of the research, they were assured that their information would remain confidential. Also, after obtaining consent from the subjects and giving them some money as reward, the measures were administered.

\section{References}

1. SeyedAlinaghi S, Taj L, Mazaheri-Tehrani E, Ahsani-Nasab S, Abedinzadeh $\mathrm{N}$, McFarland $\mathrm{W}$, et al. HIV in Iran: Onset, responses, and future directions. AIDS. 2021;35(4):529-42. doi: 10.1097/QAD.0000000000002757. [PubMed: 33252485]. [PubMed Central: PMC7924262].

2. Ministry of Health and Medical Education. Ministry of Health and Medical Education. Tehran, Iran: Ministry of Health and Medical Education; 2020, [cited 2020]. Available from: https://behdasht.gov.ir/.

3. Katibaei J. Comparison of quality of life, stress and mental health of addicts and non-addicts affected by HIV and healthy individuals. Int J Behav Sci. 2010;4(2):135-9.

4. Sadock BS. [Synopsis of psychiatry: Behavioral sciences/clinical psychiatry]. Tehran, Iran: Arjmand; 2007. Persian.

5. Nakasujja N, Skolasky RL, Musisi S, Allebeck P, Robertson K, Ronald A, et al. Depression symptoms and cognitive function among individuals with advanced HIV infection initiating HAART in Uganda. BMC Psychiatry. 2010;10:44. doi: 10.1186/1471-244X-10-44. [PubMed: 20537129]. [PubMed Central: PMC2901316].

6. Rubin LH, CookJA, Weber KM, Cohen MH, Martin E, Valcour V, et al. The association of perceived stress and verbal memory is greater in HIVinfected versus HIV-uninfected women. J Neurovirol. 2015;21(4):422-32. doi: 10.1007/s13365-015-0331-5. [PubMed: 25791344]. [PubMed Central: PMC4562210].

7. Maki PM, Rubin LH, Valcour V, Martin E, Crystal H, Young M, et al. Cognitive function in women with HIV: Findings from the Women's Interagency HIV Study. Neurology. 2015;84(3):231-40. doi: 10.1212/WNL.0000000000001151. [PubMed: 25540304]. [PubMed Central: PMC4335997].

8. Kanmogne GD, Kuate CT, Cysique LA, Fonsah JY, Eta S, Doh R, et al. HIV-associated neurocognitive disorders in sub-Saharan Africa: A pilot study in Cameroon. BMC Neurol. 2010;10:60. doi: 10.1186/1471-237710-60. [PubMed: 20626870]. [PubMed Central: PMC2912842].

9. Au A, Cheng C, Chan I, Leung P, Li P, Heaton RK. Subjective memory complaints, mood, and memory deficits among HIV/AIDS patients in Hong Kong. J Clin Exp Neuropsychol. 2008;30(3):338-48. doi: 10.1080/13803390701416189. [PubMed: 17852611].

10. Yap KH, Mohan D, Stephan BCM, Warren N, Allotey P, Reidpath DD. Effects of Subjective Memory Complaints (SMCs) and Social Capital on Self-Rated Health (SRH) in a Semirural Malaysian Population. J Aging Res. 2019;2019:9151802. doi: 10.1155/2019/9151802. [PubMed: 31093373]. [PubMed Central: PMC6481032].

11. Kamkwalala A, Hulgan T, Newhouse P. Subjective memory complaints are associated with poorer cognitive performance in adults with HIV. AIDS Care. 2017;29(5):654-9. doi: 10.1080/09540121.2016.1248348. [PubMed: 27788587]. [PubMed Central: PMC5584557].

12. Aasvik JK, Woodhouse A, Jacobsen HB, Borchgrevink PC, Stiles TC, Landro NI. Subjective memory complaints among patients on sick leave are associated with symptoms of fatigue and anxiety. Front Psychol. 2015;6:1338. doi: 10.3389/fpsyg.2015.01338. [PubMed: 26441716]. [PubMed Central: PMC4561749].

13. Fyock CA, Hampstead BM. Comparing the relationship between subjective memory complaints, objective memory performance, and medial temporal lobe volumes in patients with mild cognitive impairment. Alzheimers Dement (Amst). 2015;1(2):242-8. doi: 10.1016/j.dadm.2015.03.002. [PubMed: 26191540]. [PubMed Central: PMC4501028]. 
14. Carter SL, Rourke SB, Murji S, Shore D, Rourke BP. Cognitive complaints, depression, medical symptoms, and their association with neuropsychological functioning in HIV infection: A structural equation model analysis. Neuropsychology. 2003;17(3):410-9. doi: 10.1037/0894-4105.17.3.410. [PubMed:12959507].

15. Bassel C, Rourke SB, Halman MH, Smith ML. Working memory performance predicts subjective cognitive complaints in HIV infection. Neuropsychology. 2002;16(3):400-10. doi: 10.1037/|0894-4105.16.3.400. [PubMed: 12146687].

16. Cholewińska G, Szymańska B. Mental impairment and neurocognitive symptoms associated with HIV infection. HIV AIDS Rev. 2009;8(2):9-14. doi:10.1016/s1730-1270(10)60030-6.

17. Groth MG. [Handbook of Psychological Assessment]. Tehran, Iran: Sokhan; 2012. Persian.

18. Jafari Z, Steffen Moritz P, Zandi T, Kamrani A, Malyeri S. Psychometric properties of Persian version of the Rey Auditory-Verbal Learning Test (RAVLT) among the elderly. Iran J Psychiatry Behav Sci. 2010;16(1):56-64.

19. Chan I, Chan E, Au A, Leung P, Li P, Lee MP, et al. Subjective memory complaints of Chinese HIV-infected patients in Hong Kong: relationships with social support, depressive mood and medical symptoms. AIDS Care. 2007;19(9):1149-56. doi:10.1080/09540120701402780.
[PubMed: 18058399].

20. Sarmad Z, Bazargan A, Hejazi E. [Research methods in behavioral sciences]. Tehran, Iran: Agah Publication; 2006. Persian.

21. Hinkin $\mathrm{CH}$, van Gorp WG, Satz P, Marcotte T, Durvasula RS, Wood $S$, et al. Actual versus self-reported cognitive dysfunction in HIV-1 infection: memory-metamemory dissociations. J Clin Exp Neuropsychol. 1996;18(3):431-43. doi: 10.1080/01688639608408999. [PubMed: 8877626].

22. Papirovitz M, Gigi A. New Contemplation Upon Subjective Memory Complaints as a Self- Report Criterion for MCI Diagnosis. Curr Alzheimer Res. 2021;18(2):136-41. doi: 10.2174/1567205018666210416095437. [PubMed: 33882803].

23. Akbari H, Safari S. Conditions of experienced stigma in people living with HIV in Iran: a qualitative comparative analysis. Sociol Health Illn. 2020;42(5):1060-76. doi: 10.1111/1467-9566.13077. [PubMed: 32162344].

24. Dawes S, Suarez P, Casey CY, Cherner M, Marcotte TD, Letendre $S$, et al. Variable patterns of neuropsychological performance in HIV-1 infection. J Clin Exp Neuropsychol. 2008;30(6):613-26. doi: 10.1080/13803390701565225. [PubMed: 18608689]. [PubMed Central: PMC3092709]. 